\title{
CONJUGATE FUNCTIONS IN ORLICZ SPACES
}

\author{
ROBERT RYAN
}

1. The purpose of this paper is to prove the following results:

THEOREM 1. Let

$$
\tilde{f}(x)=-\frac{1}{\pi} \int_{0}^{\pi} \frac{f(x+t)-f(x-t)}{2 \tan (1 / 2) t} d t=\lim _{\varepsilon \rightarrow+0}\left\{-\frac{1}{\pi} \int_{\varepsilon}^{\pi}\right\} .
$$

The mapping $f \rightarrow \tilde{f}$ is a bounded mapping of an Orlicz space into itself if and only if the space is reflexive.

Beginning with the classical result by M. Riesz for the $L_{p}$ spaces [6; vol. I, p. 253] several authors have proved this theorem in one direction or the other for various special classes of Orlicz spaces. We mention in particular the papers by J. Lamperti [2] and S. Lozinski [4] and the results given in A. Zygmund's book [6; vol. II, pp. 116-118]. In our proof we use inequalities and techniques due to S. Lozinski $[3,4]$ to show that boundedness of the mapping implies that the space is reflexive. We use the theorem of Marcinkiewicz on the interpolation of operations [6; vol. II, p. 116] to prove that reflexivity implies the boundedness of $f \rightarrow \widetilde{f}$. Our results are more general than Lozinski's results since we use the definition of an Orlicz space given by A. C. Zaanen [5] which includes, for example, the space $L_{1}$.

Section 2 contains preliminary material about Orlicz spaces. In $\S 3$ we prove that boundedness implies reflexivity and in $\S 4$ we prove the converse.

2. Let $v=\varphi(u)$ be a nondecreasing real valued function defined for $u \geqq 0$. Assume that $\varphi(0)=0$, that $\varphi$ is left continuous and that $\varphi$ does not vanish identically. Let $u=\psi(v)$ be the left continuous inverse of $\varphi$. If $\lim _{u \rightarrow \infty} \varphi(u)=l$ is finite then $\psi(v)=\infty$ for $v>l$; otherwise $\psi(v)$ is finite for all $v \geqq 0$. The complementary Young's functions $\Phi$ and $\Psi$ are defined by

$$
\Phi(u)=\int_{0}^{u} \varphi(t) d t, \quad \Psi(v)=\int_{0}^{v} \psi(s) d s .
$$

$\Phi$ is an absolutely continuous convex function for $0 \leqq u<\infty$ and $\Psi$ is absolutely continuous and convex in the internal where it is finite.

Received January 16, 1963. Sponsored by the Mathematics Research Center, U. S. Army Madison, Wisconsin under Contract No.: DA-11-022-ORD-2059. 
If $\lim _{u \rightarrow \infty} \varphi(u)=\infty$ this internal is $0 \leqq v<\infty$. If $\lim _{u \rightarrow \infty} \varphi(u)=l$ is finite we say that $\Psi$ jumps to infinity at $v=l$.

$\Phi$ is said to satisfy the $A_{2}$-condition if there is a constant $k>0$ and a $u_{0} \geqq 0$ such that $\Phi(2 u) \leqq k \Phi(u)$ for $u \geqq u_{0}$. This is equivalent to satisfying the inequality $\Phi(l u) \leqq k l \Phi(u)$ for all sufficiently large $u$, where $l$ is any number greater than one (for a proof and further details see $[1 ; p .23])$.

The Orlicz space $L_{\Phi}=L_{\oplus}(0,2 \pi)$ consists, by definition, of all measurable complex functions $f$ defined on the unit circle for which $\|f\|_{\Phi}=\sup \int_{0}^{2 \pi}|f(t) g(t)| d t<\infty$, where the supremum is taken over all functions $g$ with $\int_{0}^{2 \pi} \Psi|g(t)| d t \leqq 1$. The space $L_{\Psi}$ is defined by interchanging $\Phi$ and $\Psi^{0}$. The Orlicz space $L_{\mu}$ is defined to be the set of all measurable complex functions $f$ for which

$$
\|f\|_{\mu \Phi}=\sup \int_{0}^{2 \pi}|f(t) g(t)| d t<\infty,
$$

where the supremum is taken over all $g$ with $\|g\|_{\Psi} \leqq 1 . L_{M \Psi}$ is similarly defined. The spaces $L_{\varphi}, L_{\Psi}, L_{M \Phi}$ and $L_{M \Psi}$ are all Banach spaces with their respective norms when functions equal almost everywhere are identified. The spaces $L_{\Phi}$ and $L_{\Delta \mu \varphi}$ consist of the same functions and $\|f\|_{\mu \Phi} \leqq\|f\|_{\Phi} \leqq 2\|f\|_{\mu}$. The same is true replacing $\Phi$ by $\Psi$. The space $L_{\oplus}$ is reflexive with dual space $L_{M \Psi}$ if and only if both $\Phi$ and $\Psi$ satisfy the $A_{2}$-condition.

Two Young's functions $\Phi_{1}$ and $\Phi_{2}$ are said to be equivalent $\left(\Phi_{1} \sim \Phi_{2}\right)$ if and only if there exist positive constants $k_{1}, k_{2}$, and $u_{0}$ such that $\Phi_{1}\left(k_{1} u\right) \leqq \Phi_{2}(u) \leqq \Phi_{1}\left(k_{2} u\right)$ for $u \geqq u_{0}$. It is clear that $\sim$ is an equivalence relation and that the $\Delta_{2}$-condition is an equivalence class property. If $\Phi_{1} \sim \Phi_{2}$ then $L_{\Phi_{1}}$ and $L_{\Phi_{2}}$ consist of the same functions and the norm \|\|$_{\Phi_{1}}$ and \|\|$_{\Phi_{2}}$ are equivalent. Conversely, if $L_{\Phi_{1}}$ and $L_{\Phi_{2}}$ have the same elements then $\Phi_{1} \sim \Phi_{2}[1 ;$ p. 112].

3. In this section we will show that if $f \rightarrow \tilde{f}$ is bounded then $L_{\Phi}$ is reflexive. Let $S_{n}(f)$ denote the $n$th partial sum of the Fourier series of $f$ and write $D_{n}(t)=\sin [n+(1 / 2)] t / 2 \sin (1 / 2) t$. If $\|\tilde{f}\|_{\infty} \leqq C\|f\|_{\odot}$ for all $f \in L_{\phi}$ then it follows [6; vol. I, p. 266] that $\left\|S_{n}(f)\right\|_{\Phi} \leqq A\|f\|_{\Phi}$ for all $f \in L_{\varphi}$ and all $n$, where $A$ is a positive constant independent of $n$ and $f$. Thus, the following result is ostensibly more general than the corresponding part of Theorem 1 .

THEOREM 2. If $\left\|S_{n}(f)\right\|_{\phi} \leqq A\|f\|_{\phi}$ for all $f \in L_{\phi}$ and all $n$ then $L_{\phi}$ is reflexive.

The proof of Theorem 2 uses the following two lemmas given by 
S. Lozinski in [3]. Lozinski proved these lemmas under more restrictive conditions on $\varphi$ than we have assumed. Nevertheless, Lozinski's proofs remain valid for the functions as we have defined them.

Lemma $1 . \quad(\varphi(u) / 250) \log (n / u \varphi(u)) \leqq\left\|D_{n}\right\|_{\odot}$ for $u \varphi(u) \geqq 1$.

Lemma 2. If $\left\|S_{n}(f)\right\|_{\Phi} \leqq A\|f\|_{\oplus}$ for all $f \in L_{\phi}$ and all $n$ then $\left\|D_{n}\right\|_{\Phi} \leqq 2 \pi A(n+\Phi(u)) / u$ for $0<u<\infty$.

Proof of Theorem 2. Our proof is a variation of the one given by Lozinski in [4]. From Lemmas 1 and 2 we have

$$
\varphi(v) \log \frac{n}{v \varphi(v)} \leqq k \frac{n+\Phi(u)}{u}
$$

for $v \varphi(v) \geqq 1$ and $0<u<\infty . k=2 \pi A / 250$. Our immediate aim is to show that for all sufficiently large $\lambda>1$

$$
\log \left(\frac{\lambda}{2}\right) \leqq 2 k \frac{\varphi(v)}{\varphi\left(\frac{v}{\lambda}\right)}
$$

for $v \geqq v_{0}$, where $v_{0}$ depends upon $\lambda$.

For any

$$
\lambda>1, \Phi(u)=\int_{0}^{u} \varphi(t) d t>\int_{u / \lambda}^{u} \varphi(t) d t
$$

and hence

$$
\Phi(u)>\left(u-\frac{u}{\lambda}\right) \varphi\left(\frac{u}{\lambda}\right)=(\lambda-1) \frac{u}{\lambda} \varphi\left(\frac{u}{\lambda}\right) .
$$

Thus

$$
\log \frac{(\lambda-1) n}{\Phi(v)}<\log \frac{n}{\frac{v}{\lambda} \varphi\left(\frac{v}{\lambda}\right)} .
$$

By combining (3) and (1) we see that

$$
\varphi\left(\frac{v}{\lambda}\right) \log \frac{(\lambda-1) n}{\Phi(v)} \leqq k \frac{n+\Phi(v)}{v}
$$

whenever $(v / \lambda) \varphi(v / \lambda) \geqq 1$. Let $n=[\Phi(v)]=$ greatest integer in $\Phi(v)$. Then (4) becomes

$$
\varphi\left(\frac{v}{\lambda}\right) \log \left\{(\lambda-1) \frac{[\Phi(v)]}{\Phi(v)}\right\} \leqq k \frac{[\Phi(v)]+\Phi(v)}{v} \leqq 2 k \frac{\Phi(v)}{v} .
$$


For every sufficiently large $\lambda$ there exist a $v_{0} \geqq 0$ such that for $v \geqq v_{0}$

$$
1<\frac{\lambda}{2} \leqq(\lambda-1) \frac{[\Phi(v)]}{\Phi(v)}
$$

and

$$
\frac{v}{\lambda} \varphi\left(\frac{v}{\lambda}\right) \geqq 1 .
$$

Using (5), (6) and the fact that $\Phi(v) \leqq v \varphi(v)$ we get inequality (2) for $v \geqq v_{0}$. Since $\lambda$ can be arbitrarily large (2) implies that $\lim _{u \rightarrow \infty} \varphi(u)=\infty$ and hence that $\Psi$ does not jump to infinity. We next show that $\Psi$ satisfies the $A_{2}$-condition.

Let $\lambda$ be large but fixed and write $l=(1 / 2 k) \log (\lambda / 2)$. Then (2) states that

$$
l \varphi\left(\frac{t}{\lambda}\right) \leqq \varphi(t)
$$

for $t \geqq v_{0}$. This implies, on taking inverses, that there is a number $s_{0}$ such that for $s \geqq s_{0}$

$$
\psi(s) \leqq \lambda \psi\left(\frac{s}{l}\right)
$$

Thus

$$
\int_{s_{0}}^{v} \psi(s) d s \leqq \lambda \int_{s_{0}}^{v} \psi\left(\frac{s}{l}\right) d s=\lambda l \int_{s_{0} / l}^{v / l} \psi(s) d s
$$

or

$$
\Psi(v)-\Psi\left(s_{0}\right) \leqq \lambda l\left[\Psi\left(\frac{v}{l}\right)-\Psi\left(\frac{s_{0}}{l}\right)\right] .
$$

This shows that for sufficiently large $v$

$$
\Psi(l v) \leqq 2 \lambda l \Psi(v)
$$

and hence proves that $\Psi$ satisfies the $A_{2}$-condition.

If $\left\|S_{n}(f)\right\|_{\phi} \leqq A\|f\|_{\Phi}$ for all $f \in L_{\Phi}$ then it follows that $\left\|S_{n}(g)\right\|_{\mu^{W}} \leqq$ $A\|g\|_{M^{W}}$ for all $g \in L_{M M^{T}}$ or, equivalently, that $\left\|S_{n}(g)\right\|_{\Psi} \leqq 2 A\|g\|_{\Psi}$ for all $g \in L_{\dddot{w}}$. Since we have shown that $\Psi$ does not jump to $\infty$ we can interchange the rôle of $\Phi$ and $\Psi$ in the above argument to show that $\Phi$ satisfies the $\Delta_{2}$-condition. This proves that $L_{\Phi}$ is reflexive and completes the proof of Theorem 2.

4. In this section we prove a general result about reflexive Orlicz 
spaces which combined with the classical results of M. Riesz [6; vol. I, p. 253 and p. 266] yields the unproved half of Theorem 1 as well as the converse of Theorem 2 .

THEOREM 3. Suppose that $T$ is a bounded linear operator on $L_{p}$ into $L_{p}$ for $1<p<\infty$. Then if $L_{\phi}$ is reflexive $T$ is defined and bounded on $L_{\phi}$ into $L_{\varphi}$.

Proof. The proof consists of showing that $\Phi$ can be replaced by an equivalent function $\Phi_{1}\left(\Phi \sim \Phi_{1}\right)$ such that $\Phi_{1}$ satisfies the conditions of the Marcinkiewicz theorem on the interpolation of operations i.e. such that

$$
\int_{u}^{\infty} \frac{\Phi_{1}(t)}{t^{\beta+1}} d t=O\left\{\frac{\Phi_{1}(u)}{u^{\beta}}\right\}
$$

and

$$
\int_{1}^{u} \frac{\Phi_{1}(t)}{t^{\alpha+1}} d t=O\left\{\frac{\Phi_{1}(u)}{u^{\alpha}}\right\}
$$

for $u \rightarrow \infty$, where $1<\alpha<\beta<\infty$.

The assumption that $L_{\phi}$ is reflexive implies that $\lim _{u \rightarrow \infty} \varphi(u)=\infty$ and hence that $\lim _{u \rightarrow \infty} \Phi(u) / u=\infty$. By $[1 ;$ p. 16] $\Phi$ is equal for sufficiently large values of $u$ to a function $M$ of the form $M(u)=$ $\int_{0}^{u} p(t) d t$ where $p$ is a nondecreasing right continuous function with $\lim _{u \rightarrow 0} p(u)=0$ and $\lim _{u \rightarrow \infty} p(u)=\infty$. Clearly $\Phi \sim M$.

By $\left[1 ;\right.$ p. 46] the function $M_{1}$ defined by $M_{1}(u)=\int_{0}^{u}(M(t) / t) d t$ is equivalent to $M$ and hence to $\Phi$. The derivative of $M_{1}^{0}$ is continuous and strictly increasing.

Since $L_{\phi}$ is reflexive both $\Phi$ and $\Psi$ satisfy the $A_{2}$-condition. Thus both $M_{1}$ and its conjugate Young's function $N_{1}$ satisfy the $A_{2}$-condition [1; p. 23]. According to [1; pp. 26-27] this implies the existence of numbers $a, b$, and $u_{0} \geqq 0$ with $1<a<b<\infty$ such that

$$
1<a<\frac{u M_{1}^{\prime}(u)}{M_{1}(u)}<b
$$

for all $u \geqq u_{0}$. If we define $\Phi_{1}$ by

$$
\Phi_{1}(u)= \begin{cases}\frac{M_{1}\left(u_{0}\right)}{u_{0}^{a}} u^{a} & \text { for } u \leqq u_{0} \\ M_{1}(u) & \text { for } u \geqq u_{0}\end{cases}
$$

we obtain a function $\Phi_{1} \sim \Phi$ such that 


$$
1<a \leqq \frac{u \varphi_{1}(u)}{\Phi_{1}(u)} \leqq b
$$

for all $u \geqq 0$.

We next show that $\Phi_{1}$ satisfies (12) and (13) for suitably chosen $\alpha$ and $\beta$. In particular choose $\alpha$ and $\beta$ such that $1<\alpha<a \leqq b<\beta<\infty$. This is clearly possible. In what follows all of the integrals will exist as finite numbers because of (14).

Integration by parts shows that

$$
\int_{u}^{\infty} \frac{\varphi_{1}(t)}{t^{\beta}} d t=\beta \int_{u}^{\infty} \frac{\Phi_{1}(t)}{t^{\beta+1}} d t-\frac{\Phi_{1}(u)}{u^{\beta}}
$$

and

$$
\int_{0}^{u} \frac{\varphi_{1}(t)}{t^{\alpha}} d t=\alpha \int_{0}^{u} \frac{\Phi_{1}(t)}{t^{\alpha+1}} d t+\frac{\Phi_{1}(u)}{u^{\alpha}} .
$$

From (14) we obtain

$$
\int_{u}^{\infty} \frac{\varphi_{1}(t)}{t^{\beta}} d t \leqq b \int_{u}^{\infty} \frac{\Phi_{1}(t)}{t^{\beta+1}} d t
$$

and

$$
\int_{0}^{u} \frac{\varphi_{1}(t)}{t^{\alpha}} d t \geqq a \int_{0}^{u} \frac{\Phi_{1}(t)}{t^{\alpha+1}} d t .
$$

Combining (15) with (17) and (16) with (18) shows that

$$
\int_{u}^{\infty} \frac{\Phi_{1}(t)}{t^{\beta+1}} d t \leqq \frac{1}{\beta-b}\left\{\frac{\Phi_{1}(u)}{u^{\beta}}\right\}
$$

and

$$
\int_{0}^{u} \frac{\Phi_{1}(t)}{t^{\alpha+1}} d t \leqq \frac{1}{a-\alpha}\left\{\frac{\Phi_{1}(u)}{u^{\alpha}}\right\} .
$$

This shows that $\Phi_{1}$ satisfies (12) and (13). Thus by the Marcinkiewicz theorem and Theorem 10.14 of [6; vol I, p. 174] there exists a constant $K_{1}$ such that $\|T f\|_{\Phi_{1}} \leqq K_{1}\|f\|_{\Phi_{1}}$ for all $f \in L_{\Phi_{1}}$. Since $\Phi \sim \Phi_{1}$ there is a constant $K$ such that $\|T f\|_{\odot} \leqq K\|f\|_{\Phi}$ for all $f \in L_{\oplus}$. This completes the proof of Theorem 3.

Statements of the standard corollaries of Theorem 1 can be found in [2].

\section{REFERENCES}

1. M. A. Krasnosel'skii and Ya. B. Rutickii, Convex Functions and Orlicz Spaces, Groningen, 1961. 
2. J. Lamperti, A note on conjugate functions, Proc. Amer. Math. Soc., 10 (1959), 71-76.

3. S. Lozinski, On convergence and summability of Fouries series and interpolation processes, Mat. Sbornik N.S., 14 (1944) 175-262.

4. - On convergence in mean of Fourier series Doklady, 51 (1949), 7-10.

5. A. C. Zaanen, Linear Analysis, New York-Amsterdam-Groningen, 1953.

6. A. Zygmund, Trigonometric Series, Vols. I, II, Cambridge, 1959. 
